\title{
IL-4 induces M2 macrophages to produce sustained analgesia via opioids
}

\author{
Melih Ö. Celik, 'Dominika Labuz, ${ }^{1} J a c q u e l i n e ~ K e y e,{ }^{2}$ Rainer Glauben, ${ }^{2}$ and Halina Machelska' \\ 'Department of Experimental Anesthesiology and ${ }^{2}$ Medizinische Klinik für Gastroenterologie, Infektiologie und \\ Rheumatologie, Charité - Universitätsmedizin Berlin, corporate member of Freie Universität Berlin, Humboldt-Universität \\ zu Berlin, and Berlin Institute of Health, Campus Benjamin Franklin, Berlin, Germany.
}

IL-4 is a pleiotropic antiinflammatory cytokine, which can be neuroprotective after nervous system injury. The beneficial actions of IL- 4 are thought to result from the blunting of action of inflammatory mediators, such as proinflammatory cytokines. Here, we demonstrate that IL-4 induces M2 macrophages to continuously produce opioid peptides and ameliorate pain. IL-4 application at injured nerves in mice shifted $\mathrm{F} 4 / 80^{+}$macrophages from the proinflammatory $\mathrm{M} 1$ to the antiinflammatory M2 phenotype, which synthesized opioid peptides (Met-enkephalin, $\boldsymbol{\beta}$-endorphin, and dynorphin A 1-17). These effects were accompanied by a long-lasting attenuation of neuropathy-induced mechanical hypersensitivity, beyond the IL-4 treatment. This IL-4induced analgesia was decreased by opioid peptide antibodies and opioid receptor $(\delta, \mu, \kappa)$ antagonists applied at injured nerves, which confirms the involvement of the local opioid system. The participation of M2 macrophages was supported by analgesia in recipient mice injected at injured nerves with $\mathrm{F} 4 / 80^{+}$macrophages from IL-4-treated donors. Together, IL-4-induced M2 macrophages at injured nerves produced opioid peptides, which activated peripheral opioid receptors to diminish pain. Fostering the opioid-mediated actions of intrinsic M2 macrophages may be a strategy to tackle pathological pain.

Authorship note: MÖC and DL contributed equally to this work.

Conflict of interest: The authors have declared that no conflict of interest exists.

Copyright: (c) 2020, American Society for Clinical Investigation.

Submitted: August 30, 2019

Accepted: January 22, 2020

Published: February 27, 2020.

Reference information: JCI Insight. 2020;5(4):e133093.

https://doi.org/10.1172/jci.

insight.133093.

\section{Introduction}

IL-4 is an antiinflammatory cytokine acting as a pleiotropic regulator of numerous immune and inflammatory processes. It is typically secreted by T helper 2 lymphocytes, mast cells, eosinophils, and basophils and plays a protective role in neurological disorders (e.g., multiple sclerosis, spinal cord injury). The beneficial actions of IL- 4 are considered to result from the inhibition of the production and release of proinflammatory cytokines, chemokines, proteases, and reactive oxygen species $(1,2)$. Earlier studies reported analgesic actions of IL- 4 to be mediated by dampening the proinflammatory cytokine response in animal models (3-5). Importantly, pathological pain, such as pain resulting from nerve injury, is associated with neuroinflammation, as immune cells, including macrophages, accumulate at the damaged nerves. Current research predominately focuses on the contribution of these cells to pain pathogenesis (6-8). However, macrophages are functionally diverse and comprise various subtypes, including classically activated proinflammatory M1 and alternatively activated antiinflammatory M2 populations. M1 macrophages are induced by proinflammatory cytokines and bacterial lipopolysaccharides and secrete various proinflammatory mediators, IL-1 $\beta, \mathrm{TNF}$, and nitric oxide $(9,10)$, which activate sensory neurons and exacerbate pain $(6,8,11)$. In contrast, M2 macrophages release low amounts of proinflammatory molecules but higher levels of antiinflammatory mediators, such as IL-10. Furthermore, IL-4 is critical for the skewing of macrophages toward an M2 phenotype $(2,10)$, which was suggested to attenuate pain by elevating IL-10 expression in animals (12).

Notably, immune cells, such as lymphocytes, neutrophils, and macrophages, contain opioid peptides, Met-enkephalin (ENK), $\beta$-endorphin (END), and dynorphin A 1-17 (DYN), which upon release reduce pain in animal models and in humans (13-18). Additionally, we have recently shown that opioid peptides are secreted by IL-4-polarized M2 macrophages in vitro (19).

In this study, we demonstrate that repetitive IL-4 application at the damaged nerves produced long-lasting analgesia via endogenous opioids in a mouse model of neuropathy-induced pathological pain. Specifcally, IL-4 locally induced M2 macrophages to produce opioid peptides, which via activation of peripheral 
opioid receptors attenuated neuropathy-triggered mechanical hypersensitivity. The analgesic effects persisted for several days after cessation of IL-4 treatment. Our results suggest that the endogenous opioid system is crucial to the action of IL-4 and M2 macrophages in pain control.

\section{Results}

IL-4 application at injured nerves attenuates neuropathy-induced mechanical but not heat hypersensitivity. As a model of pathological pain, we used a chronic constriction injury (CCI) of the sciatic nerve, which represents human peripheral neuropathy due to nerve entrapment or compression $(16,18,20)$. The CCI resulted in profound, lasting up to 26 days mechanical hypersensitivity (manifested by reduced thresholds to von Frey filaments; Figure 1A) and heat hypersensitivity (demonstrated by shortened withdrawal latencies, assessed by the Hargreaves test; Supplemental Figure 1; supplemental material available online with this article; https://doi.org/10.1172/jci.insight.133093DS1). These effects occurred in hind paws innervated by damaged nerves (Figure 1A and Supplemental Figure 1) but not in uninjured contralateral paws $(P>0.05$; Supplemental Figure 1 and Supplemental Figure 2A).

To access the effects of IL-4, we injected IL-4 (200 ng) at the damaged nerve (CCI site) daily, starting from day 14 until day 21 after CCI. This treatment did not modify heat hypersensitivity (Supplemental Figure 1) but attenuated mechanical hypersensitivity (Figure 1A). Thus, IL-4 produced short-lasting analgesia (5-15 minutes) after the first 5 injections (until day 18 after CCI), which then persisted for 24 hours after the next 3 injections (until day 22), and was further maintained after cessation of IL-4 injection, for the following 4 days (until day 26). Vehicle administered at the CCI site did not alter ipsilateral paw withdrawal thresholds (Figure 1A) and latencies (Supplemental Figure 1), and there were no changes in the contralateral paws after any treatment $(P>0.05$; Supplemental Figure 1 and Supplemental Figure 2A).

To determine whether IL-4 receptors (IL-4R) contribute to the long-lasting IL-4-induced (200 ng) analgesia, we used IL-4R $\alpha$ blocking antibody (anti-IL-4R $\alpha ; 6 \mu \mathrm{g}$ ). We found that IL-4-induced analgesia was diminished by anti-IL-4R $\alpha$ injected with the last IL-4 application (on day 21 after CCI). However, antiIL-4R $\alpha$ was without effect when injected later, on days 22 and 26 after CCI, when IL-4-induced analgesia remained despite no further IL-4 injections (Figure 1B). There were no changes in the contralateral paws after any treatment $(P>0.05$; Supplemental Figure $2 \mathrm{~B})$. These data suggest that IL-4R $\alpha$ are required for the direct action of IL- 4 but are not involved in the absence of exogenous IL- 4 and imply that persistent IL-4induced analgesia involves additional mechanisms. In the following experiments, we tested the hypothesis that IL-4 shifts macrophages toward an M2 phenotype and stimulates them to produce opioid peptides, which results in the continuous alleviation of pain.

IL-4 treatment predominantly increases macrophage counts at injured nerves. To analyze the effect of IL-4 on the immune status of injured nerves, we first quantified immune cells using flow cytometry. We performed this analysis 24 hours after the last (eighth) injection of IL-4 (200 ng; on day 22 after CCI), when the persistent IL-4-induced analgesia was fully established (Figure 1A); we also used this schedule for all ex vivo experiments in the following experiments. We found that injured nerves were infiltrated by $\mathrm{CD} 45^{+}$ cells, including $\mathrm{CD}^{+} \mathrm{T}$ lymphocytes, $\mathrm{Ly}^{+} \mathrm{g}^{+}$neutrophils, and $\mathrm{F} 4 / 80^{+}$macrophages, in both control and IL-4-treated animals (Figure 2A). However, as quantitative analysis revealed, IL-4 elevated the number of $\mathrm{CD} 45^{+}$cells as well as all 3 leukocyte populations. The strongest effect was seen for macrophages, which represented a 3 -fold increase in cell count compared with the vehicle (Figure 2B).

IL-4 treatment shifts macrophages at injured nerves toward an M2 phenotype. To examine the inflammatory status of macrophages, we isolated them from injured nerves by $\mathrm{F} 4 / 80^{+}$immunomagnetic separation (IMS) and analyzed by the real-time quantitative reverse transcription PCR (qRT-PCR) for the mRNA

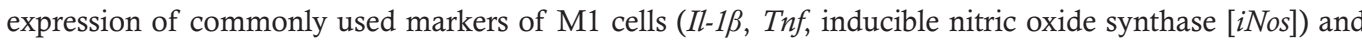
M2 cells (Il-10, arginase-1 [Arg-1], chitinase-like 3 protein [Ym1]) (1, 9). Using flow cytometry, we confirmed the purity of sorted $\mathrm{F} 4 / 80^{+}$macrophages by showing that they constituted $94 \%-96 \%$ of all separated cells (Supplemental Figure 3).

The F4/80+ cells collected on day 22 after CCI from control animals (24 hours after the last vehicle injection) expressed substantially higher mRNA levels of M1 than M2 cell markers (Figure 3), suggesting that damaged nerves were infiltrated by proinflammatory M1 macrophages. Importantly, IL-4 (200 ng) treatment resulted in diminished mRNA levels of M1 cell markers and in elevated mRNA levels of M2 cell markers, analyzed 24 hours after the last IL-4 injection (on day 22 after CCI) (Figure 4). These data indicate that IL-4 shifted macrophages in injured nerves from the M1 to the antiinflammatory M2 status. 

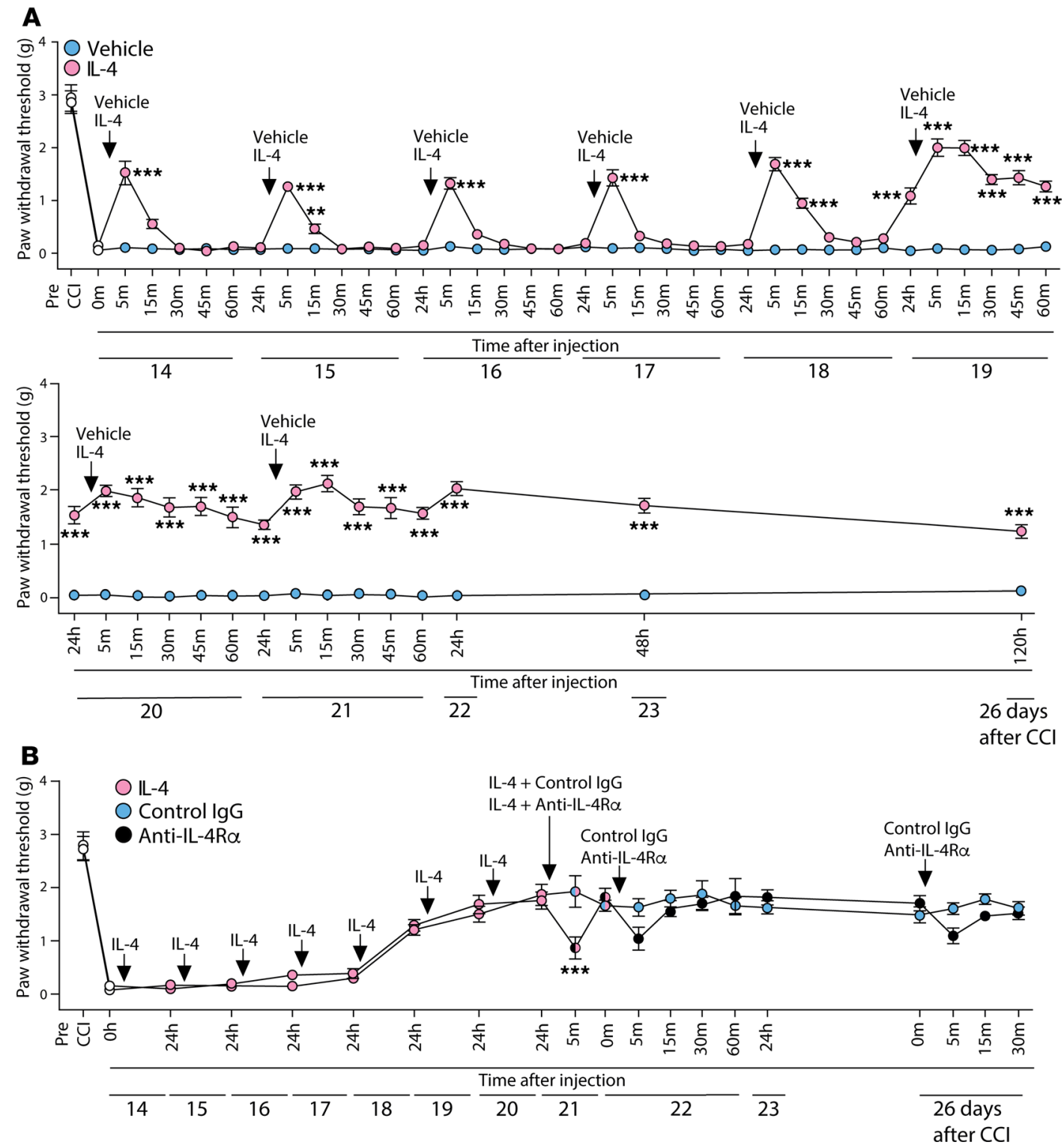

Figure 1. IL-4 application at damaged nerves produces long-lasting attenuation of mechanical hypersensitivity. (A) Time course of IL-4-induced analgesia. IL-4 (200 ng) was injected daily on days 14-21 after chronic constriction injury (CCI) at the CCI site. Mechanical von Frey thresholds were measured before, 5-60 minutes after, and 24 hours after each injection until day 22 and then on day 23 (48 hours after the last injection) and on day 26 after CCI (120 hours after the last injection). (B) Involvement of IL-4R in IL-4-induced analgesia. Anti-IL-4R $\alpha(6 \mu \mathrm{g})$ was injected with IL-4 (200 ng) on day 21 after CCI (when IL-4 was applied last time), and again alone (without IL-4) on days 22 and 26 after CCI. von Frey thresholds were measured before and 24 hours after each IL-4 injection (on days 14-21); 5 minutes after IL-4 and anti-IL-4R $\alpha$ co-injection (on day 21); before and 5-60 minutes after (on day 22) and 24 hours after (on day 23) the second anti-IL-4R $\alpha$ injection; and before and 5-30 minutes after the third anti-IL-4R $\alpha$ injection (on day 26). The thresholds were measured in hind paws ipsilateral to CCl. Control groups were tested accordingly. Arrows indicate injections. ${ }^{* *} P<0.01,{ }^{* * *} P<0.001$ vs. control (vehicle or control lgC); 2-way repeated-measures ANOVA and Bonferroni's test. Data are represented as mean \pm SEM. $n=9$ animals per group.

IL-4-induced M2 macrophages at damaged nerves produce opioid peptides. Next, we investigated whether IL-4 (200 ng) treatment influences the production of opioid peptides in macrophages. We analyzed the F4 $/ 80^{+}$-sorted macrophages from injured nerves by qRT-PCR to quantify the mRNA of opioid peptide precursors, proenkephalin (Penk), proopiomelanocortin (Pomc), and prodynorphin (Pdyn) (Figure 5A). We also measured the content of the corresponding opioid peptides, ENK, END, and DYN in these cells by enzyme immunoassays (Figure 5B). We found that macrophages isolated on day 22 after CCI from control animals (24 hours after the last vehicle injection) expressed low levels of opioid peptide precursor mRNAs and contained low amounts of opioid peptides. In contrast, after IL-4 treatment, both the precursor mRNAs and the intracellular opioid peptide contents were substantially elevated 
A
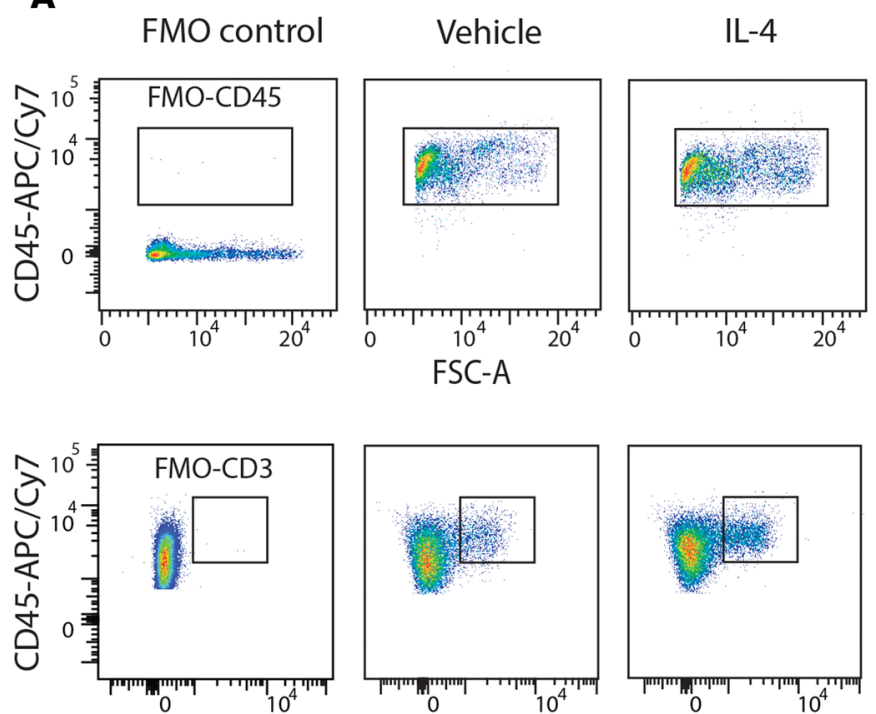

CD3-APC
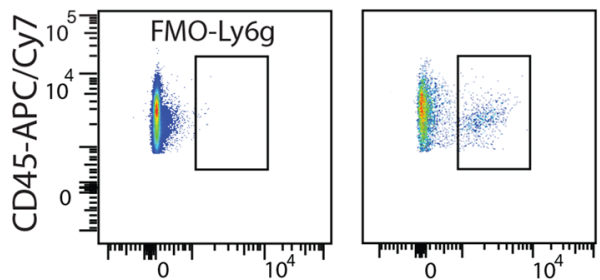

Ly6g-FITC
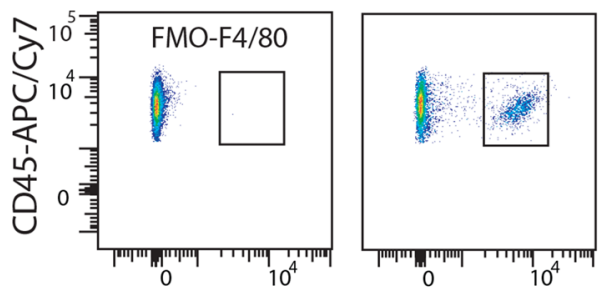

F4/80-PE
B

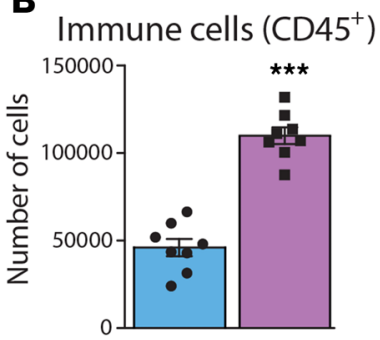

T lymphocytes $\left(\mathrm{CD}^{+}\right)$

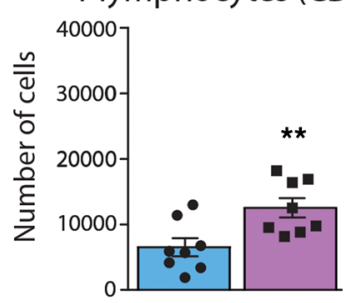

Neutrophils $\left(\mathrm{Ly} \mathrm{g} \mathrm{g}^{+}\right)$

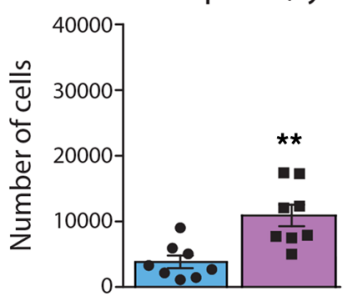

Macrophages $\left(\mathrm{F} 4 / 80^{+}\right)$

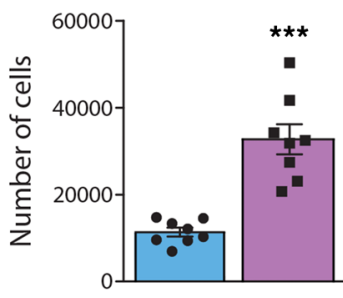

Vehicle IL-4

Figure 2. IL-4 treatment primarily increases macrophage numbers at injured nerves. (A) Representative dot plots

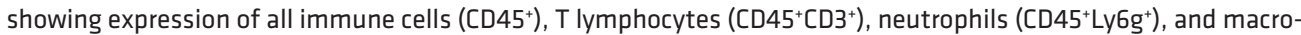
phages $\left(C D 45^{+} \mathrm{F} 4 / 80^{+}\right)$. The blots represent the corresponding fluorescence minus one (FMO) negative controls (left), cells isolated from injured nerves after repetitive injection of vehicle (middle) or IL-4 (200 ng; right). Cells stained positive for the appropriate marker are shown inside the rectangular gates. (B) Quantification of the corresponding cell populations shown in A. ${ }^{* *} P<0.01,{ }^{* *} P<0.001$ vs. vehicle; 2 -tailed $t$ test. Data are shown as individual data points and mean \pm SEM. $n=8$ samples per group. The cells were isolated 24 hours after the last injection of vehicle or IL-4, on day 22 after chronic constriction injury. The data were analyzed using flow cytometry and Flowjo software. The number of positively stained cells were calculated using absolute counting beads.

(Figure 5). Although, as stated in Methods, we did not exclude any animals or samples from the statistical analysis, Grubbs' test identified a significant outlier $(P<0.05)$ in the DYN content data in the IL-4 group (Figure 5B). Nevertheless, the exclusion of this outlier did not change the outcome of these data because the DYN level remained significantly enhanced in the IL-4 group $(0.049 \pm 0.008 \mathrm{ng} / \mathrm{mL}$, $n=7)$ compared with the control group $(0.013 \pm 0.003 \mathrm{ng} / \mathrm{mL}, n=8 ; P<0.001$, 2-tailed $t$ test $)$.

To find out which macrophage population produces opioid peptides after IL-4 treatment, we performed FISH by RNAscope in F4/80'-sorted macrophages. We used probes to Il-1 $\beta$ and $A r g-1$ as representative markers of M1 and M2 macrophages, respectively, and to Penk as a representative opioid peptide precursor. The cellular localization of all 3 mRNAs was confirmed by their presence in the vicinity of the nuclear 


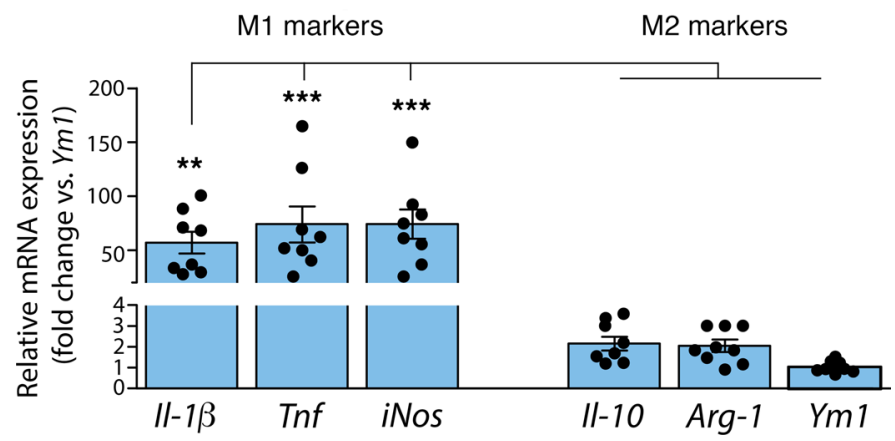

Figure 3. Injured nerves are infiltrated by proinflammatory M1 macrophages. Quantitative mRNA expression of M1 markers (II-1 $\beta$, Tnf, iNos) and M2 markers (II-10, Arg-1, Ym1) in F4/80+ macrophages isolated by IMS from injured nerves of control animals (24 hours after the last vehicle injection at the chronic constriction injury [CCI] site) on day 22 after $\mathrm{CCl} .{ }^{* *} P<0.01,{ }^{* *} P<0.001$ represent a significant difference between the respective M1 marker and each M2 marker; 1-way repeated-measures ANOVA and Bonferroni's test. Data were acquired by qRT-PCR, represent relative mRNA expression levels normalized to Gapdh, are expressed as fold change vs. $Y m 1$, and are shown as individual data points and mean \pm SEM. $n=8$ samples per group.

marker DAPI (Figure 6A). Quantitative analysis revealed that IL-4 treatment decreased the percentage of M1 cells (expressing Il-1 $1 \beta$ mRNA) and elevated the percentages of M2 cells (expressing Arg-1 mRNA) and cells expressing Penk mRNA (Figure 6B), in line with the data in Figures 4 and 5. Additionally, after both IL-4 and vehicle treatment, there was a very low percentage of cells coexpressing Il-1 $\beta$ and $\mathrm{Arg}-1$ mRNAs, confirming that these markers indeed stain distinct macrophage populations. Importantly, there was no difference between control and IL-4 groups in the percentage of macrophages coexpressing Il-1 $\beta$ and Penk mRNAs, but IL-4 significantly increased the percentage of macrophages coexpressing $A r g-1$ and Penk mRNAs (Figure 6C). Collectively, these data suggest that cells producing opioid peptides in response to IL-4 treatment are M2 macrophages.

IL-4-induced M2 macrophages produce analgesia. To directly determine the effects of M2 versus M1 macrophages on CCI-induced mechanical hypersensitivity, we isolated $\mathrm{F} 4 / 80^{+}$macrophages by IMS from injured nerves of donor mice treated with vehicle or IL-4 (200 ng; on day 22 after CCI, i.e., 24 hours after the last injections) and transferred them $\left(10^{5}\right.$ cells $)$ at the injured nerves of recipient mice (on days 22 and 23 after CCI). We found that macrophages from IL-4-treated mice diminished mechanical hypersensitivity for 15 minutes after the first injection (on day 22) in recipient mice. This analgesic effect lasted up to 90 minutes after the second macrophage injection (on day 23). In contrast, the transfer of macrophages from vehicle-treated donors did not alter mechanical hypersensitivity after any injection in recipient mice (Figure 7). These findings confirm that M2 macrophages account for IL-4-induced analgesia.

IL-4-induced analgesia is mediated by opioid peptides and receptors at injured nerves. The results described above demonstrate that repetitive IL-4 treatment polarized macrophages to an M2 phenotype, that these cells contained higher levels of opioid peptides than M1 cells, and produced analgesia. Here, we investigated whether this analgesia involved the endogenous opioid system, including opioid peptides (ENK, END, DYN) and opioid receptors $(\delta, \mu, \kappa)$. Opioid peptide antibodies and opioid receptor antagonists were injected at the CCI site 24 hours after the last IL-4 (200 ng) application (on day 22 after CCI), when IL-4-induced ongoing analgesia was fully established, as well as 5 days after the last IL-4 injection (on day 26 after CCI), when analgesia remained despite no further IL-4 application. We found that selective blocking antibodies to ENK $(2 \mu \mathrm{g})$, END $(2 \mu \mathrm{g})$, or DYN $(4 \mu \mathrm{g})$ decreased analgesic actions of IL-4 at both time points (Figure 8A). This analgesia was also diminished by a peripherally restricted opioid receptor antagonist naloxone methiodide (NLXM; $10 \mu \mathrm{g}$ ) (Figure 8B), and by selective antagonists of $\delta$ ( $N, N$-diallyl-Tyr-Aib-Aib-Phe-Leu [ICI 174,864]; $8 \mu \mathrm{g}$ ), $\mu$ (D-Phe-Cys-TyrD-Trp-Orn-Thr-Pen-Thr-NH2 [CTOP]; $2 \mu \mathrm{g}$ ), and $\kappa$ receptors (nor-binaltorphimine [norBNI]; $2 \mu \mathrm{g}$ ) (Figure $8 \mathrm{C}$ ). The withdrawal thresholds in contralateral paws were not significantly changed by any of the treatments $(P>0.05$; Supplemental Figure 4$)$. These findings indicate that IL-4-induced analgesia is constantly mediated by endogenous opioid peptides acting at opioid receptors in injured nerves. 


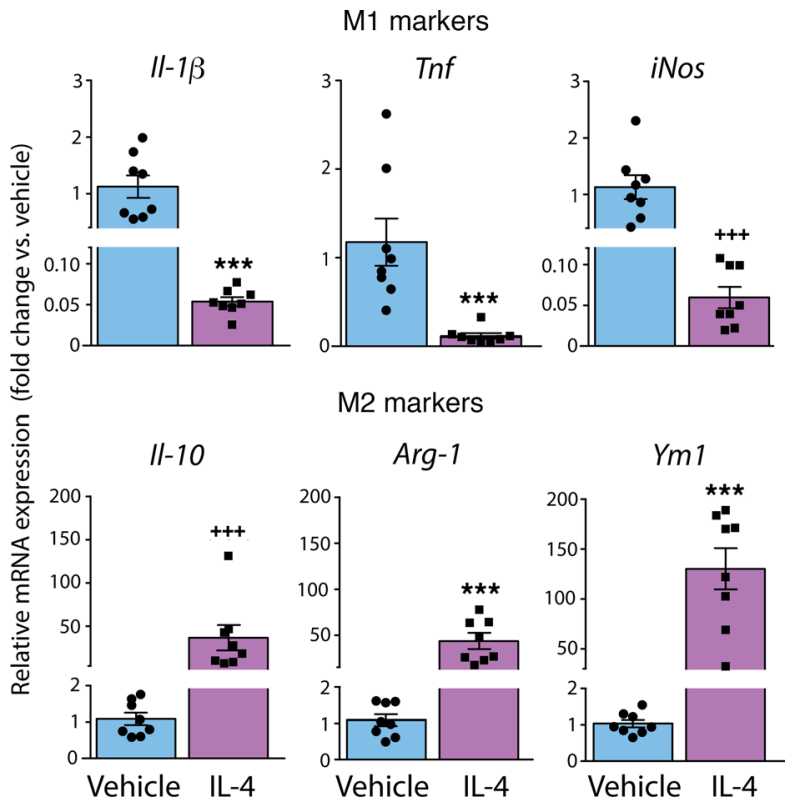

Figure 4. IL-4 treatment shifts macrophages at injured nerves into an M2 phenotype. Quantitative mRNA expression of M1 markers (II-1 $\beta$, Tnf, iNos) and M2 markers (II-10, Arg-1, Ym1) in F4/80+ macrophages isolated by IMS from injured nerves 24 hours after the last injection of vehicle or IL-4 $(200 \mathrm{ng})$ at the chronic constriction injury (CCI) site, on day 22 after CCI. ${ }^{* * *} P<0.001$ (2-tailed $t$ test), ${ }^{+++} P<0.001$ (Mann-Whitney $U$ test) vs. vehicle. Data were acquired by qRT-PCR, represent relative mRNA expression levels normalized to Gapdh, are expressed as fold change vs. vehicle, and are shown as individual data points and mean \pm SEM. $n=8$ samples per group.

\section{Discussion}

Our main finding in this study is that IL-4-induced M2 macrophages continuously produced opioids to relieve pain. Hence, IL-4 repetitively applied at the injured peripheral nerve shifted macrophages from the M1 to M2 phenotype, which produced opioid peptides (ENK, END, DYN). The opioids activated peripheral opioid receptors $(\delta, \mu, \kappa)$ and ameliorated nerve injury-triggered mechanical hypersensitivity, beyond the discontinuation of IL- 4 treatment. Specifically, IL-4 primarily increased F4/80+ macrophage counts at damaged nerves, and these cells expressed low mRNA levels of proinflammatory markers (Il-1 $\beta$, Tnf, $i$ Nos) and enhanced mRNA levels of antiinflammatory markers (Il-10, Arg-1, Ym1), supporting their M2 status. Concurrently, IL-4-induced macrophages expressed elevated levels of opioid peptides (ENK, END, DYN) and mRNAs of their precursors (Penk, Pomc, Pdyn). Single-cell analysis revealed the higher percentage of macrophages coexpressing Arg-1 and Penk mRNAs after IL-4 treatment, proving that opioids were produced by M2 cells. Adoptive transfer of these cells diminished mechanical hypersensitivity in recipient mice, directly showing their analgesic actions. Persistent IL-4-induced analgesia was indeed opioid-dependent, since it was abolished by opioid peptide antibodies and opioid receptor antagonists.

Upon injury, blood monocyte-derived macrophages are recruited to the damaged tissue, and by secretion of proinflammatory mediators, including proinflammatory cytokines, contribute to the generation of pain ( 7 , 21-23). Accordingly, we detected higher mRNA levels of proinflammatory mediators (Il-1 $\beta$, Tnf, iNos) than antiinflammatory markers (Il-10, $\mathrm{Arg}-1, \mathrm{Ym} 1)$ in $\mathrm{F} 4 / 80^{+}$macrophages isolated from injured nerves of control (vehicle-treated) mice (on day 22 after CCI), indicating their M1 status. Concomitantly, we measured low levels of opioid peptides (ENK, END, DYN) and mRNAs of their precursors (Penk, Pomc, Pdyn). Thus, as all these effects correlated with the CCI-induced hypersensitivity, not only classical pro/antiinflammatory molecule imbalance, but also endogenous opioid deficiency in macrophages, appear relevant to pain pathogenesis. Hence, strategies predominately based on dampening the proinflammatory properties of macrophages might be insufficient to inhibit pain. This is supported by a modest decrease or no changes in hypersensitivity after general depletion of macrophages in models of neuropathic, inflammatory, and postoperative pain (24-28).

Therefore, our approach in this study was to promote the analgesic properties of macrophages. We took advantage of IL-4 to polarize macrophages into an antiinflammatory M2 state $(2,10,29,30)$. We used IL-4 as a therapeutic agent and did not aim to mimic its endogenous levels, since they are apparently 
A

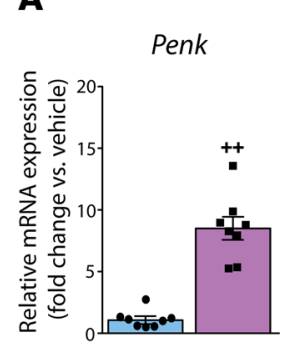

B
Precursor mRNA

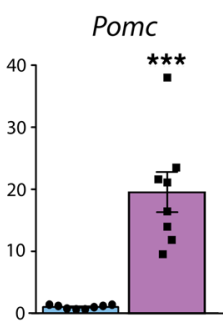

Peptide content

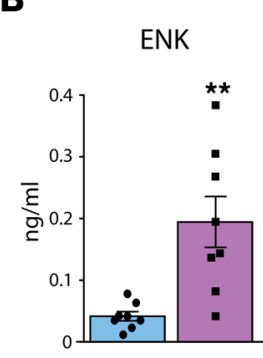

END
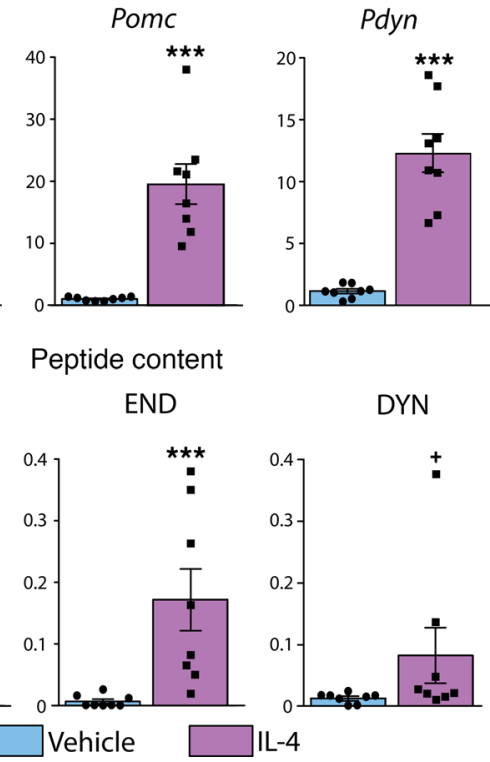

Figure 5. IL-4-induced macrophages at damaged nerves produce opioid peptides. (A) Quantitative mRNA expression of opioid peptide precursors Penk, Pomc, and Pdyn in F4/80+ macrophages from injured nerves. Data were acquired by qRT-PCR, represent relative mRNA expression levels normalized to Gapdh and are expressed as fold change vs. vehicle. (B) Intracellular content of opioid peptides ENK, END, and DYN in $\mathrm{F} 4 / 80^{+}$macrophages from injured nerves, measured by enzyme immunoassays. $\mathrm{F} 4 / 80^{+}$macrophages were isolated by IMS from nerves 24 hours after the last injection of vehicle or IL-4 (200 ng) at the chronic constriction injury (CCI) site, on day 22 after $\mathrm{CCl} .{ }^{+} P<0.05,{ }^{++} P<0.01$ (Mann-Whitney $U$ test), ${ }^{* *} P<0.01,{ }^{* * *} P<0.001$ (2-tailed $t$ test) vs. vehicle. Data are shown as individual data points and mean \pm SEM. $n=8$ samples per group. ENK, Met-enkephalin; END, $\beta$-endorphin; DYN, dynorphin A 1-17.

insufficient to resolve pain, as the CCI-triggered hypersensitivity persisted in the control (vehicle-treated) group throughout the whole time course, up to day 26 after CCI. Using flow cytometry, we found that IL-4 elevated numbers of immune cells at the injured nerves, which could result from the IL-induced immune cell extravasation and/or proliferation (31-33). Importantly, F4/80 macrophages were the predominant cell population after IL-4 treatment. Previous studies found a correlation between IL-4-induced analgesia and decreased expression of Il-1 $\beta$ and Tnf, or increased expression of IL-10 and M2 markers (Arg-1, Cd206). However, these cytokines and markers were measured in cultured peritoneal cells $(3,4)$, spinal cord homogenates (5), and macrophage cell line- or in vitro-cultured sciatic nerve fragments (12). Clearly, these conditions do not reflect the in vivo IL-4 effects, and macrophages were not directly examined in those studies. In contrast, we have tested fresh, uncultured $\mathrm{F} 4 / 80^{+}$macrophages isolated by IMS from injured nerves and found that after in vivo IL-4 treatment, they downregulated mRNAs of M1 markers (Il-1 $\beta$, Tnf, iNos) and upregulated mRNAs of M2 markers (Il-10, Arg-1, Ym1). Importantly, these M2 macrophages synthesized higher amounts of opioid peptides than M1 cells. This was evidenced by elevated mRNA levels of opioid peptide precursors (Penk, Pomc, Pdyn) measured by qRT-PCR and confirmed by single-cell FISH, which showed Penk and Arg-1 mRNA coexpression. The M2 macrophages also contained elevated levels of ENK, END, and DYN proteins determined by enzyme immunoassays. A typical pathway of IL-4-induced M2 macrophage polarization involves activation of the transcription factor signal transducer and activator of transcription 6, which enhances transcription of M2-associated genes and decreases transcription of M1-associated genes $(1,2,9)$. It will thus be interesting to examine whether this pathway is also involved in the IL-4-induced upregulation of opioid peptides in M2 macrophages.

Furthermore, the F4 $/ 80^{+}$macrophages isolated from injured nerves of IL-4-treated, but not vehicle-treated, donor mice diminished mechanical hypersensitivity after adoptive transfer at damaged nerves in recipient mice, clearly showing that M2 cells account for IL-4-induced analgesia. The longer-lasting analgesia after the second macrophage injection is in line with our previous study, which showed that after the first injection of in vitro-polarized M2 macrophages, only small proportion of cells remained at the nerves of donor mice, possibly due to their removal by endogenous cells, and the second injection was more 
A

Vehicle

DAPI

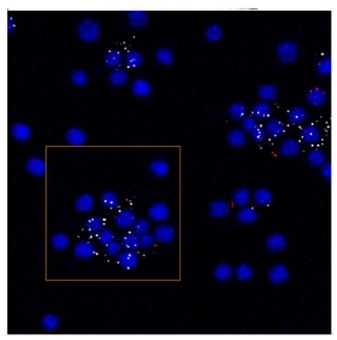

$\operatorname{Arg}-1$

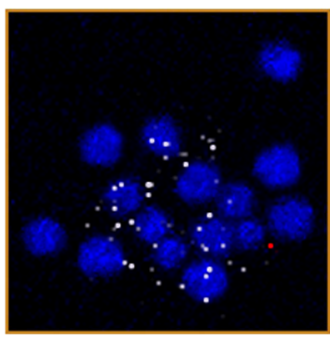

DAPI
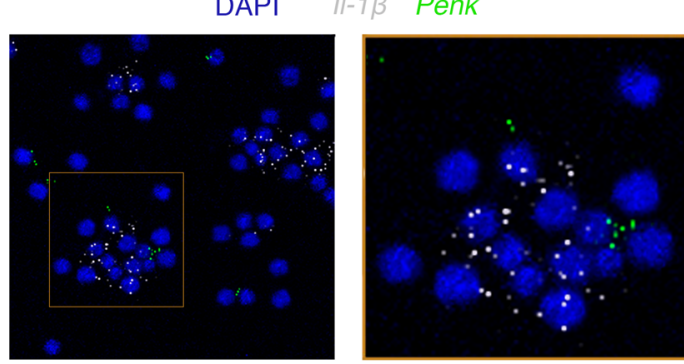

DAPI Arg-1 Penk
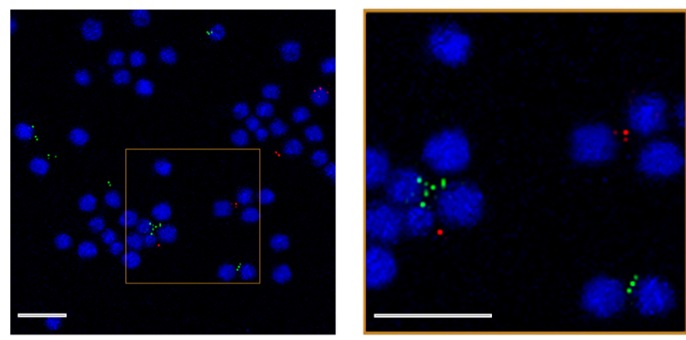

B

$\|-1 \beta$

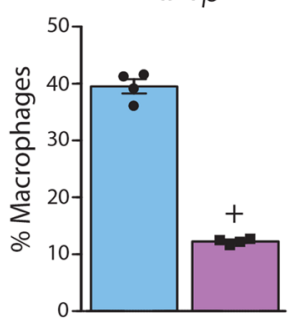

C

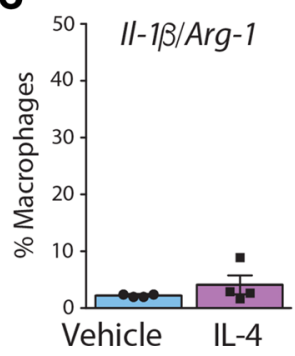

Arg-1

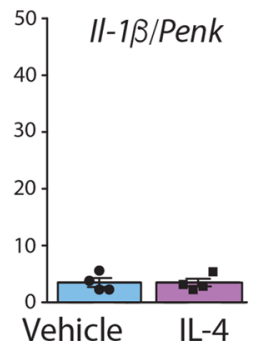

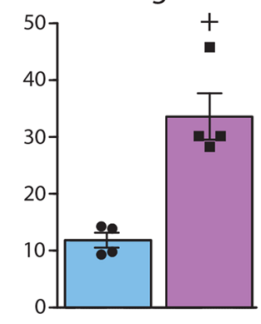

IL-4

DAPI $\quad \|-1 \beta \quad \operatorname{Arg}-1$
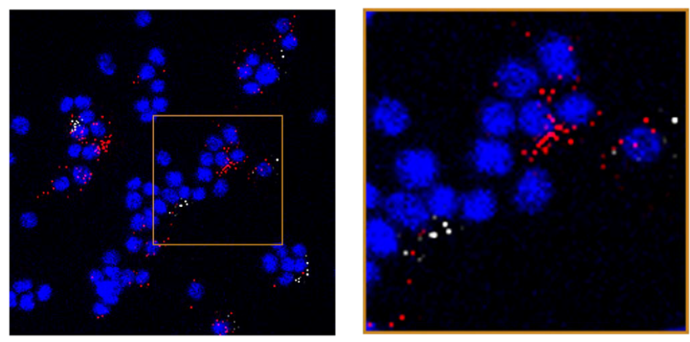

DAPI
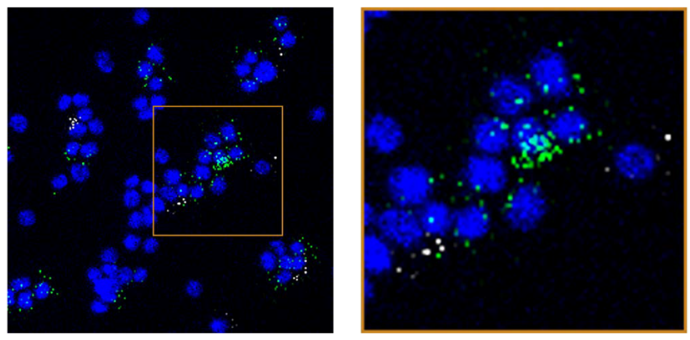

DAPI Arg-1 Penk
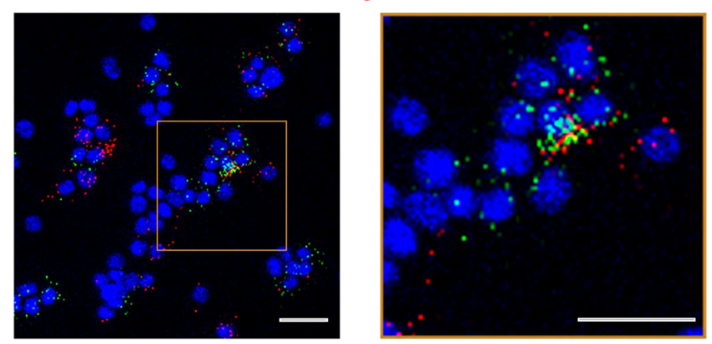

Penk
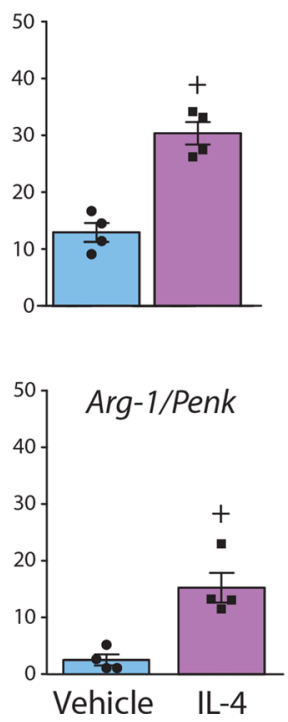

Figure 6. IL-4-induced macrophages expressing Penk mRNA are $\mathbf{M} \mathbf{2}$ macrophages. (A) Representative FISH images showing expression of mRNAs of $I I-1 \beta$ and Arg-1, $I I-1 \beta$ and Penk, and Arg- 1 and Penk in $\mathrm{F} 4 / 80^{+}$macrophages with DAPI-stained nuclei. The cells were isolated from injured nerves of mice treated with vehicle (left panel) or IL-4 (200 ng; right panel). In each image set, the bigger orange marked square (on the right-hand side) represents a higher magnification of the corresponding square in the image on the left-hand side. In each panel, the left (vehicle) and the right (IL-4), the 3 images on the left-hand side show the same field of view taken from the same sample under different staining conditions. Scale bars: $20 \mu \mathrm{m}$. (B) Percentage of $\mathrm{F} 4 / 80^{+}$macrophages expressing mRNA of $\|-1 \beta$, Arg-1, or Penk. (C) Percentage of F4/80+ macrophages coexpressing mRNAs of $I I-1 \beta$ and Arg-1, $I I-1 \beta$ and Penk, or Arg-1 and Penk. F4/80+ macrophages were isolated by IMS from injured nerves 24 hours after the last injection of vehicle or IL-4 (200 ng) at the chronic constriction injury (CCI) site, on day 22 after $\mathrm{CCl} .{ }^{+} P<0.05$ vs. vehicle; Mann-Whitney $U$ test. Data are shown as individual data points and mean \pm SEM. $n=$ 4 samples per group. 


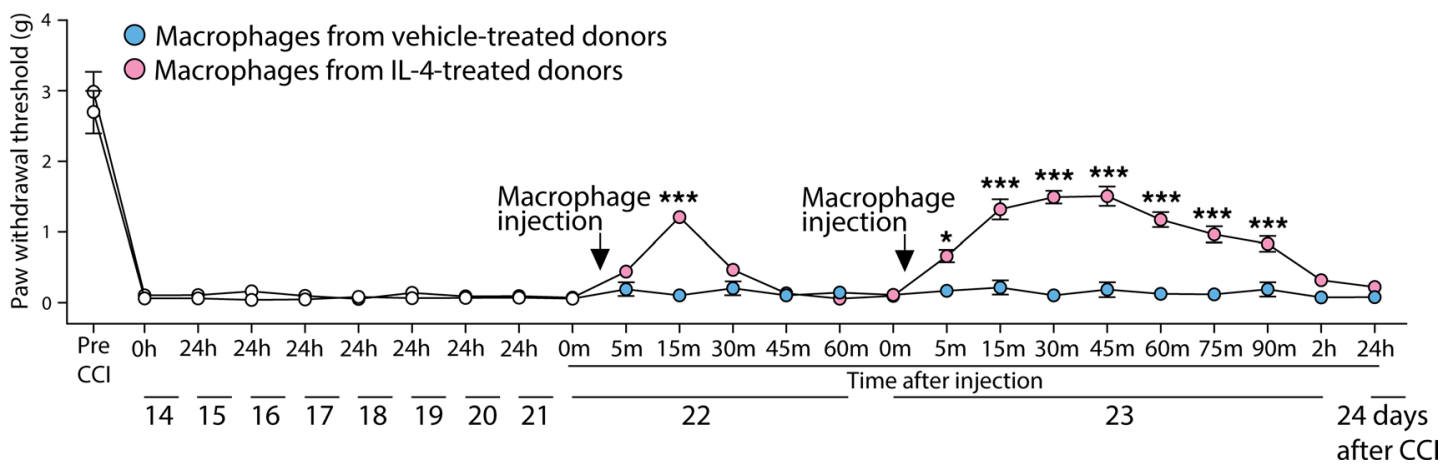

Figure 7. IL-4-induced M2 macrophages produce analgesia. F4/80+ macrophages isolated by IMS from injured nerves of donor mice treated with vehicle or IL-4 (200 ng; on day 22 after chronic constriction injury [CCI], i.e., 24 hours after the last injection) were adoptively transferred ( $10^{5}$ cells) twice (indicated by arrows) at injured nerves (CCI site) of recipient mice (on days 22 and 23 after CCI). von Frey thresholds were measured once a day (every 24 hours) until day 21 (without any injections); before and 5-60 minutes after the first macrophage injection (on day 22); before and 5 minutes to 2 hours after the second macrophage injection (on day 23); and 24 hours later (on day 24). The thresholds were measured in hind paws ipsilateral to $C \mathrm{Cl}$. ${ }^{*} P<0.05,{ }^{* * *} P<0.001$, recipients $(n=6)$ injected with macrophages from IL-4-treated donors vs. recipients $(n=3)$ injected with macrophages from vehicle-treated donors; 2 -way repeated-measures ANOVA and Bonferroni's test. Data are represented as mean \pm SEM.

efficient (19). Moreover, the reduction of IL-4-induced analgesia by opioid peptide antibodies and opioid receptor antagonists applied at the CCI site clearly demonstrates that the local endogenous opioid system is essential for pain control by IL-4 and M2 macrophages. All 3 opioid peptides (ENK, END, DYN) and receptors $(\delta, \mu, \kappa)$ are involved, since the selective blockade of each was equally effective. Additionally, the inhibition of IL-4-induced analgesia by NLXM, an opioid receptor antagonist with limited blood-brain barrier permeability (34), suggests that the effects were mediated by peripheral opioid receptors. This has important implications because in contrast to opioid receptors in the brain, the activation of peripheral opioid receptors is devoid of serious side effects such as respiratory arrest, sedation, and addiction $(35,36)$. Interestingly, all these opioid effects occurred both 24 hours and 5 days after discontinuation of IL- 4 treatment; of note, the analgesia was equally efficient and did not diminish even at the latter time point. Since this persistent analgesia was not attenuated by IL-4R $\alpha$ antibody, it was apparently independent of IL-4R $\alpha$, regardless whether they are expressed on immune cells or neurons (37-39), but appears to involve constitutive release of opioid peptides from M2 macrophages. This is in line with our earlier in vitro experiments, in which extracellular levels of opioid peptides were measured (18 hours) after the removal of IL- 4 from the medium (19). Conversely, the short-lasting (5 minutes), IL-4-induced analgesia clearly involved IL-4R $\alpha$, since it was diminished by IL-4R $\alpha$ antibody coinjected with IL-4 (day 21 after CCI). This suggests an acute direct action of IL-4 via IL-4R $\alpha$, possibly involving the release of opioids, which will be addressed in a follow-up study. These findings suggest that when macrophages are polarized by IL-4 into the M2 phenotype, they can continuously use opioids to ameliorate nerve injury-triggered mechanical pain.

The involvement of $\delta$-, $\mu$ - and $\kappa$-opioid receptors at the CCI site in analgesia, mediated by IL-4-induced M2 macrophages, is supported by studies showing that all 3 receptors were detected in sensory fibers (16) and $\mu$ and $\delta$ receptors were upregulated (40-42) at the nerve injury site. The CCI predominantly results in the degeneration of $\mathrm{A} \beta$ fibers (43), and thus the main remaining sensory fibers are $\mathrm{C}$ and $\mathrm{A} \delta$ fibers. Both fiber types express opioid receptors (44-47) and transmit mechanical and heat stimuli $(48,49)$. Consistently, exogenous opioid receptor agonists applied at the CCI site attenuated mechanical and heat hypersensitivity $(40,46,50-52)$. However, here we found that endogenous opioid peptides, derived from IL-4-induced M2 macrophages, attenuated mechanical but not heat hypersensitivity. This is in agreement with our previous study, in which heat hypersensitivity was attenuated by perineurally applied exogenous opioids, but not by opioid peptides, including ENK, END, and DYN (53). Even though both exogenous and endogenous opioids activate the same opioid receptors, it is possible that these ligands initiate different downstream mechanisms to interact with various pain modality-dependent ion channels or intracellular pathways $(36,53)$, and further studies are required. Interestingly, other studies found that M2 macrophages polarized by morphine or peroxisome proliferator-activated receptor- $\gamma$ agonist reduced mechanical but not heat hypersensitivity in models of inflammatory and postoperative pain $(28,54)$. Additionally, IL-4-knockout mice displayed enhanced, acute mechanical but unaltered heat sensitivity (55), although the reasons for these differences were not provided. Thus, as the role of immune cells in the modulation of different pain modalities 

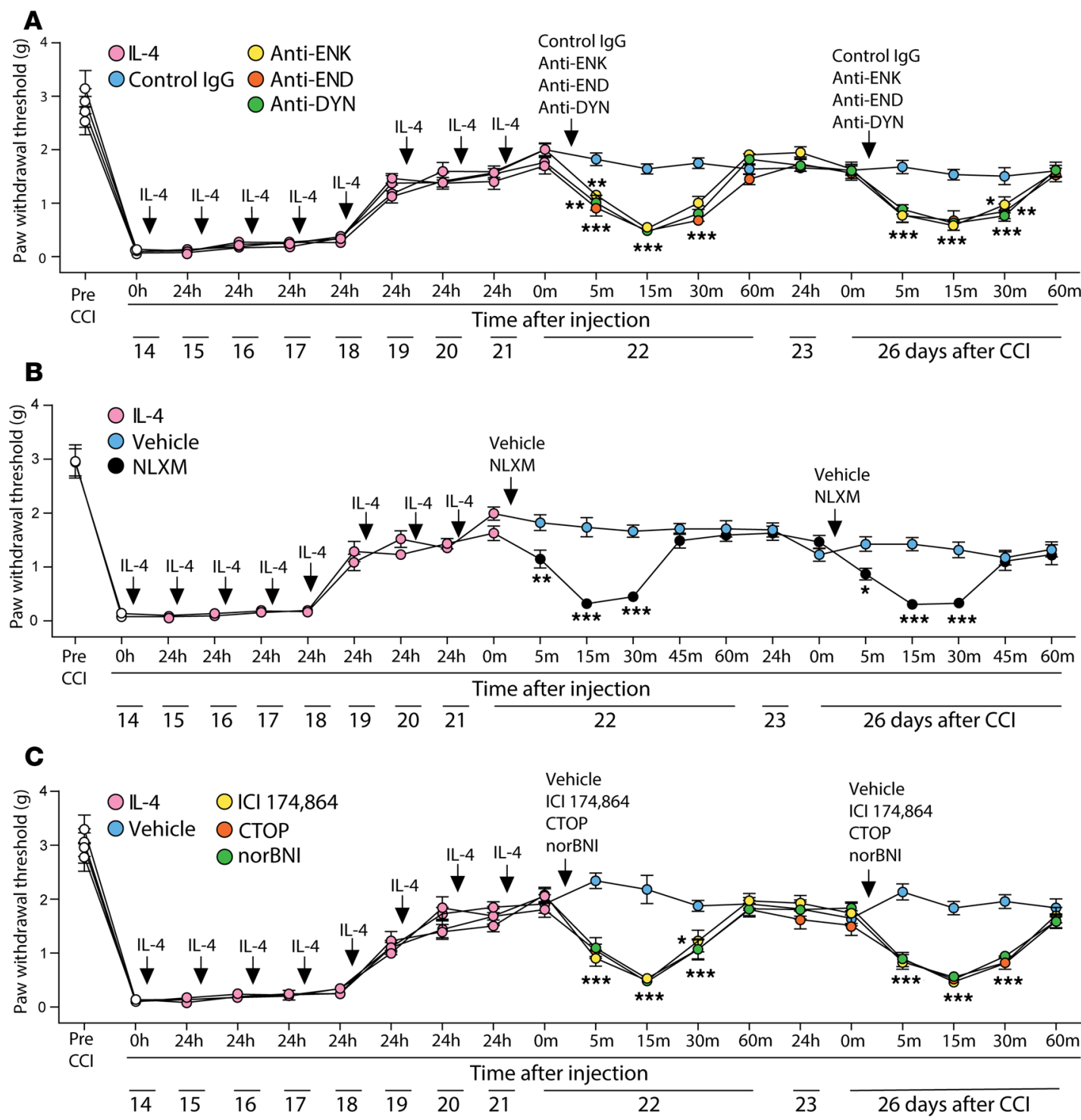

Figure 8. Sustained IL-4-induced analgesia is mediated by opioid peptides and receptors at damaged nerves. (A) Reversibility of IL-4-induced (200 ng) analgesia by antibodies to opioid peptides, anti-ENK $(2 \mu \mathrm{g})$, anti-END $(2 \mu \mathrm{g})$, or anti-DYN (4 $\mu \mathrm{g})$. (B) Reversibility of IL-4-induced (200 ng) analgesia by peripherally restricted opioid receptor antagonist NLXM $(10 \mu \mathrm{g})$. (C) Reversibility of IL-4-induced ( $200 \mathrm{ng})$ analgesia by antagonists selective at $\delta$ (ICI 174,864; $8 \mu \mathrm{g}$ ), $\mu$ (CTOP; $2 \mu \mathrm{g}$ ), and $\kappa$ receptors (norBNI; $20 \mu \mathrm{g}$ ). Antibodies and antagonists were injected 24 hours after the last IL-4 injection (on day 22 after chronic constriction injury [CCI]) and again 5 days after IL-4 injection (on day 26 after CCI). von Frey thresholds were determined before and 24 hours after each IL-4 injection (on days 14-21); before and 5-60 minutes after the first injection of antibodies and antagonists (on day 22 ); once on day 23 (24 hours after the first injection of antibodies and antagonists); and before and 5-60 minutes after the second injection of antibodies and antagonists (on day 26). The thresholds were measured in hind paws ipsilateral to $\mathrm{CCl}$. Control groups were tested accordingly. Arrows indicate injections. ${ }^{*} P<0.05,{ }^{* *} P<0.01,{ }^{* * *} P<0.001 \mathrm{vs}$. control (control IgG or vehicle); 2-way repeated-measures ANOVA, Bonferroni's test. Data are represented as mean \pm SEM. $n=9$ animals per group. ENK, Met-enkephalin; END, $\beta$-endorphin; DYN, dynorphin A 1-17; NLXM, naloxone methiodide; ICI 174,864, N,N-diallyl-Tyr-Aib-Aib-Phe-Leu; CTOP, D-Phe-Cys-TyrD-Trp-Orn-Thr-Pen-Thr-NH2; norBNI, nor-binaltorphimine.

remains to be clarified, it appears that opioid-dependent actions of IL-4 and M2 macrophages are mainly beneficial in diminishing mechanical hypersensitivity. Because we used male mice, it will also be important to examine females, since the involvement of macrophages in pain may be sex dependent (56).

In conclusion, in this project we explored pain-inhibiting actions of macrophages in response to IL-4 treatment. This is a timely area of research because the lack of and the need for such studies have been increasingly recognized $(8,57)$. We propose that fostering the beneficial effects of intrinsic M2 macrophages is more promising than the general inhibition of neuroinflammation for tackling pathological pain. The actions of IL- 4 are believed to be mediated primarily by the inhibition of proinflammatory mediators $(2,58)$. Yet, here we provide evidence that the endogenous opioid system is essential to the actions of 
IL-4 and M2 macrophages in pain control. Interestingly, IL-4 blood levels were reduced in patients with chronic widespread pain (including fibromyalgia and complex regional pain syndrome type 1) compared with healthy volunteers (59) and tended to be lower in patients with painful peripheral neuropathies than patients with painless peripheral neuropathies (60). It might thus be interesting to test IL-4 for pain treatment in clinical trials. Notably, IL-4 therapy (for 6 weeks or 3 months) was well tolerated and diminished inflammation in patients with psoriasis $(61,62)$. Together, our findings may have wide implications, since IL-4 and M2 cells play a role in inflammatory and neurodegenerative diseases $(2,9,10,63)$. The opioid system may be particularly beneficial in peripheral neuroinflammatory conditions, since the activation of peripheral opioid receptors can provide analgesia without centrally mediated adverse effects $(35,36)$. Nonetheless, our study does not preclude the search for strategies targeting other macrophage-derived mediators, including antiinflammatory cytokines and specialized proresolving mediators.

\section{Methods}

Sample size. Statistical power calculations to estimate animal/sample numbers per group were performed using the $G^{*}$ Power 3.1.2 program. In the in vivo experiments, each group comprised 9 animals and were tested on 2-3 different days. Exceptions were adoptive cell transfer experiments (Figure 7), in which 3 and 6 recipient mice in control and IL-4 groups, respectively, were used and tested on 2 different days. This was necessary to keep the animal numbers to a minimum because relatively high numbers of donor mice were needed (see below).

In the ex vivo experiments, each group comprised 8 independent biological samples and were measured on 2-3 different days. Exceptions were FISH experiments (Figure 6), in which 4 independent biological samples per group were used and analyzed on 2 different days. This was needed to keep the animal numbers to a minimum because, in this method, a relatively high number of cells are initially needed due to a considerable number of cells being lost during the challenging and long-lasting procedure. Besides, additional cells/animals are required for controls (see also below). The exact numbers of animals and samples per group are given in the figure legends.

Randomization and blinding. Animals were randomly placed in cages by an animal caretaker who was not involved in the study. In vivo experiments were performed by an experimenter blinded to the treatments. Substances or cells were prepared in separate, coded vials (one per animal) by a colleague not involved in the in vivo testing, and the codes were broken after completion of experiments. Similarly, flow cytometry experiments and quantification of FISH data were performed by an experimenter unaware of the sample assignment. A colleague not involved in these experiments coded flow cytometry samples and FISH slides; the codes were broken after completion of the analyses.

Animals. Male C57BL/6J mice (22-30 g, 6-13 weeks old, Janvier Laboratories) were kept in groups of 2-4 per cage, with free access to food and water, in an environmentally controlled room (12 hours light/ dark schedule, light on at $7 \mathrm{am} ; 22^{\circ} \mathrm{C} \pm 0.5^{\circ} \mathrm{C}$; humidity $60 \%-65 \%$ ). The experiments were carried out during the light cycle. After completion of the in vivo experiments and for tissue collection for the ex vivo experiments, animals were killed with isoflurane overdose (AbbVie). All efforts were made to minimize animal suffering and to reduce their number (see above).

Induction of neuropathy. CCI was induced in deeply isoflurane-anesthetized mice. The sciatic nerve was exposed at the level of the right mid-thigh and 3 loose silk ligatures (4/0) were placed around the nerve with $1-\mathrm{mm}$ spacing. The ligatures were tied until they elicited a brief twitch in the right hind limb. The wound was closed with silk sutures and animals were monitored during recovery from the anesthesia $(18,19,51)$.

Assessment of mechanical sensitivity (von Frey test). Animals were habituated to the test cages (IITC Life Sciences, model 410) daily (1-2 times for 15 minutes), starting 6 days before nociceptive testing. To assess mechanosensitivity, calibrated von Frey filaments (Stoelting) in the range of $0.054 \mathrm{mN}(0.0056 \mathrm{~g})$ to 42.85 $\mathrm{mN}$ (4.37 g) were used. The filaments were applied until they bowed, for approximately 3 seconds, to the plantar surface of hind paws. The up-down method was used to estimate $50 \%$ withdrawal thresholds (64). Testing began using a $2.74 \mathrm{mN}(0.28 \mathrm{~g})$ filament. In the absence of withdrawal, the next stronger filament was applied. If the animal withdrew the paw, the preceding weaker filament was applied. The maximum number of applications was 6-9, and the cutoff was $42.85 \mathrm{mN}(4.37 \mathrm{~g})$. The sequence of paws was alternated between animals to avoid "order" effects $(18,19,51)$.

Assessment of heat sensitivity (Hargreaves test). Mice were habituated to the test cages (IITC Life Sciences, model 336) before the testing, as described above for the von Frey test. To examine heat sensitivity, radiant 
heat was applied to the plantar surface of hind paws from underneath the glass floor with a high-intensity projector lamp bulb and paw withdrawal latency was evaluated using an electronic timer. The withdrawal latency was defined as the average of 2 measurements separated by at least 10 seconds. The heat intensity was adjusted to obtain baseline withdrawal latency of about 10-12 seconds in uninjured paws, and the cutoff was set at 20 seconds to avoid tissue damage. The sequence of paws was alternated between animals to avoid "order" effects $(19,51)$.

In vivo treatments. Injections were performed perineurally at the site of nerve injury (CCI site, $30 \mu 1)$ under brief isoflurane anesthesia. A polyethylene tube was placed $2 \mathrm{~mm}$ from the tip around the needle to ensure the same depth of needle insertion into the middle of the scar after CCI, as described earlier $(16,18,51)$.

To assess the time course of IL-4-induced analgesia, the recombinant mouse IL-4 (200 ng, R\&D Systems) was injected daily, beginning on day 14 until day 21 after CCI. To examine the contribution of IL-4R to IL-4-induced analgesia, anti-IL-4R $\alpha$ (6 $\mu \mathrm{g}, \mathrm{BD}$ Pharmingen, clone mIL-4R-M1) and isotype-matched control IgG2ak (6 $\mu$ g, BD Pharmingen) were injected with IL-4 (200 ng) on day 21 after CCI (when IL-4 was applied last time), and again alone (without IL-4) on days 22 and 26 after CCI. To evaluate the contribution of opioid peptides and opioid receptors to IL-4-induced analgesia, we used anti-END ( $2 \mu \mathrm{g}$, Peninsula Laboratories, catalog T-4044), anti-ENK ( $2 \mu \mathrm{g}$, Peninsula Laboratories, catalog T-4293), anti-DYN (4 $\mu \mathrm{g}$, Peninsula Laboratories, catalog T-4267), control rabbit IgG (4 $\mu \mathrm{g}$, Sigma-Aldrich), NLXM (10 $\mu \mathrm{g}$, Sigma-Aldrich), CTOP (2 $\mu \mathrm{g}$, Sigma-Aldrich), norBNI (2 $\mu \mathrm{g}$, Sigma-Aldrich), and ICI 174,864 (8 $\mu \mathrm{g}$, Biozol). They were injected 24 hours after the last IL-4 injection (on day 22 after CCI) and again 5 days after the last IL-4 injection (on day 26 after CCI).

For adoptive cell transfer experiments, $\mathrm{F} 4 / 80^{+}$macrophages were isolated by IMS (see below) from injured nerves of donor mice treated with vehicle or IL-4 $(200 \mathrm{ng}$; on day 22 after CCI, i.e., 24 hours after the last injections) and were injected ( $10^{5}$ cells) twice at injured nerves (CCI site) of recipient mice (on days 22 and 23 after CCI). Cells pooled from 5 vehicle-treated donor mice or from 3 IL-4-treated donor mice were needed for one recipient mouse. Mechanical and heat sensitivity were measured according to the schedules depicted in Figure 1, Figure 7, Figure 8, and Supplemental Figures 1, 2, and 4, and are described in the corresponding figure legends.

All doses/cell numbers were chosen based on pilot experiments. IL- 4 was dissolved in $0.1 \%$ BSA (Sigma-Aldrich) in PBS (Biochrom). Cells were suspended in PBS, and all other substances were dissolved in sterile water and diluted with $0.9 \% \mathrm{NaCl}$. Control groups were treated with the respective solvents.

Immune cell isolation from injured nerves. Based on the in vivo experiments, all below-described ex vivo experiments were performed 24 hours after the last (eighth) IL-4 (200 ng) injection (CCI site) on day 22 after CCI, when long-lasting IL-4-induced analgesia was fully established.

Mice were perfused transcardially with ice-cold PBS under terminal isoflurane anesthesia, and the ligated part of the sciatic nerve (approximately $1-\mathrm{cm}$ long), including the ligation site and sites distal and proximal to it, was isolated. The nerves were cut into small pieces, digested, filtered, and centrifuged, and the cell pellet was resuspended in RPMI 1640 with GlutaMAX buffer (Gibco) (18). The cells were stained with acridine orange/propidium iodide stain (Logos) to verify their viability; counted using Luna dual fluorescence cell counter; and immediately used for further experiments.

Flow cytometry. Two injured sciatic nerve fragments were pooled for each of 8 replicates, and single-cell suspensions were prepared as described above. Each replicate was sufficient to quantify all cell populations (Figure 2). All experiments followed the guidelines for the use of flow cytometry and cell sorting in immunological studies (65). The isolated immune cells were first labeled with LIVE/DEAD Fixable Aqua Dead Cell Stain Kit, for 30 minutes on ice, according to the manufacturer's instructions (Thermo Fisher Scientific), to exclude dead cells. The cells were washed with ice-cold PBS containing $2 \%$ FBS (Biochrom) and centrifuged, and the supernatant was removed. Cells were stained for 10 minutes on ice with anti-mouse CD16/32 (Fc Block, clone 93, BioLegend) to ensure antigen-specific binding. To identify immune cell populations, the cells were labeled with anti-mouse CD45-APC-eFluor 780 (clone 30 F11, eBioscience), CD3-APC (clone 17A2, eBioscience), Ly-6G-FITC (clone 1A8, BioLegend), and F4/80-PE (clone BM8, BioLegend). All antibodies were prepared in PBS containing 2\% FBS. As staining controls, the fluorescence minus one, single-stain, unstain, and all-stain controls were included for accurate identification of immune cells, compensation, and voltage adjustments. To quantify the absolute number of cells, $50 \mu$ l CountBright absolute counting beads (Thermo Fisher Scientific) were added to the solutions according to the manufacturer's recommendations before the flow cytometer analysis $(66,67)$. 
Counting beads were gated on the forward scatter- versus linear side-scatter plot. The percentages of positively stained cells determined over 10,000 events were analyzed using FACS Canto II (BD Biosciences), and fluorescence intensity was expressed in arbitrary units on a logarithmic scale. Single cells were gated on the forward scatter-height versus forward scatter-area density plot to exclude doublets and the data were analyzed using FlowJo software (version 10.1r5, Tree Star Inc.) (19).

IMS of macrophages. $\mathrm{F} 4 / 80^{+}$macrophages were separated from immune cells isolated from injured nerve fragments (pooled from 3 animals) using EasySep Release Mouse PE Positive Selection Kit (StemCell) according to the manufacturer's protocol. Briefly, immune cells were labeled with F4/80-PE antibody (clone BM8, BioLegend) for 20 minutes and magnetic particles (StemCell) for 10 minutes and then separated using an EasySep Magnet (StemCell). Negatively stained F4/80- cells were poured off, whereas the positively stained $\mathrm{F} 4 / 80^{+}$cells remained in the tube. Then, to acquire particle-free cells, bound magnetic particles were removed from the isolated $\mathrm{F} 4 / 80^{+}$cells using the release buffer (StemCell). The purity of F4/80 $0^{+}$separated cells ( $\sim 10^{5}$ cells) was verified by flow cytometry, as described above. The separated macrophages were immediately used for the adoptive transfer (see above) and all experiments described below.

$q R T-P C R$. For qRT-PCR experiments, 3 injured nerve fragments were pooled for each of 8 replicates. From each replicate, the $\mathrm{F} 4 / 80^{+}$macrophages were isolated by IMS (see above), and from these cells the total RNA was extracted and used to measure mRNA levels of all M1 and M2 markers and opioid peptide precursors (Figure 3, Figure 4, and Figure 5A). The isolated $\mathrm{F} 4 / 80^{+}$macrophages were disrupted and homogenized in Buffer RLT (QIAGEN) and kept at $-80^{\circ} \mathrm{C}$ until further use. The total RNA was extracted from the cells using the RNeasy Micro Kit (QIAGEN) following the manufacturer's protocol with on-column DNAse treatment. Quantity and quality of the total RNA were assessed by the DS-11 Spectrophotometer (DeNovix) and agarose gel electrophoresis (18S/28S rRNA). cDNA synthesis was done using Superscript IV VILO Master Mix (Thermo Fisher Scientific) according to the manufacturer's protocol.

The qPCR was performed in duplicates using the TaqMan Fast Advanced Master Mix (Thermo Fisher Scientific) according to the manufacturer's instructions. The following TaqMan Gene Expression

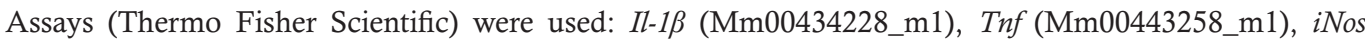
(Mm00440502_m1), Il-10 (Mm01288386_m1), Arg-1 (Mm00475988_m1), Ym1 (Mm00657889_mH), Penk (Mm01212875_m1), Pomc (Mm00435874_m1), Pdyn (Mm00457573_m1), and Gapdh (Mm99999915_g1). Additional no-RT controls were run to test for impurities or genomic DNA contaminations. The amplification was carried out for 40 cycles and the mean Ct values of the genes of interest (Il-1 $\beta$, Tnf, iNos, Il-10, Arg1, Ym1, Penk, Pomc, Pdyn) and the housekeeping gene Gapdh were calculated. The Ct values of the genes of interest were then normalized using the Ct value of the Gapdh of the same sample, and these normalized values were referred to as the $\Delta \mathrm{Ct}$ values. The $\Delta \mathrm{Ct}$ values were then used to compute the relative expression levels (i.e., fold change) of the genes of interest using the $2^{-\Delta \Delta C t}$ formula (18).

Enzyme immunoassays. For enzyme immunoassay experiments, 3 injured nerve fragments were pooled for each of 8 replicates. From each replicate, the number of $\mathrm{F} 4 / 80^{+}$macrophages isolated by IMS (see above) was sufficient to determine the intracellular content of all opioid peptides (ENK, END, DYN) (Figure 5B). The measurements were done according to the manufacturer's instructions using the kits for the corresponding opioids, ENK (Peninsula Laboratories, catalog S-1419), END (Phoenix Pharmaceuticals, catalog EK-022-06), and DYN (Phoenix Pharmaceuticals, catalog EK-021-03).The samples were thawed, resuspended in RPMI buffer (GIBCO), lysed by a freezing/thawing procedure ( $8 \mathrm{~min}$ at $-80^{\circ} \mathrm{C}$ and $1 \mathrm{~min}$ at $50^{\circ} \mathrm{C}$; repeated 5 times) followed by sonication (Ultra-Turrax T8; IKA Labortechnik). The supernatants were incubated for 2 hours with biotinylated peptide and anti-ENK, anti-END, or anti-DYN. Streptavidin-HRP was added and the samples were incubated for 1 hour. After washing, tetramethylbenzidine was added, and after 1 hour of incubation, the reaction was terminated by application of $2 \mathrm{~N} \mathrm{HCl}$. The absorbance was measured at $450 \mathrm{~nm}$ (Molecular Devices Spectra Max), according to the standard curve. All samples were measured in duplicates (18).

FISH by RNAscope. For RNAscope experiments, 3 injured sciatic nerve fragments were pooled for each of 4 replicates. From each replicate, the numbers of IMS isolated F4/80+ macrophages (see above) were sufficient for the quantification of macrophage percentages in Figure 6, B and C. RNAscope assessment (Advanced Cell Diagnostic) was performed following the manufacturer's protocols (68). Briefly, F4/80+ IMS macrophages were fixed with 10\% neutral buffered formalin (AppliChem) for 1 hour at $37^{\circ} \mathrm{C}$, centrifuged, washed with 2\% FBS in PBS (Biochrom), and incubated in 70\% EtOH overnight. Then, the cells were cytospinned using Cytospin 3 on Cytoträger slides (Shandon) and covered with Cell-Tak Cell and Tissue Adhesive 
Solution (Corning). The slides were first air dried for 20 minutes, then treated for 5 minutes with 50\%, 70\%, and $100 \% \mathrm{EtOH}$ and kept in $100 \% \mathrm{EtOH}$ overnight. Next, a hydrophobic barrier was drawn around the cells on the slides using ImmEdge Pen (Vector) and hydrogen peroxide (Advanced Cell Diagnostic) was applied. After 10 minutes of incubation at room temperature, the slides were washed twice in PBS and the cells were permeabilized using Protease III (Advanced Cell Diagnostic) in the HybEZ Oven (Advanced Cell Diagnostic) at $40^{\circ} \mathrm{C}$ for 30 minutes. Then, the cells were hybridized in the $\mathrm{HybEZ}$ oven at $40^{\circ} \mathrm{C}$ for 2 hours using mRNA probes (Advanced Cell Diagnostic), mm-Penk-C1, mm-Il-1 $\beta-\mathrm{C} 2$, and mm-Arg-1-C3. Additional negative and positive control slides were prepared, in which cells were hybridized using 3-plex ${ }^{-}$and 3-plex ${ }^{+}$control_Mm probes (Advanced Cell Diagnostic), on each experimental day. All slides were kept in $5 \times$ saline-sodium citrate buffer (Merck) overnight. Thereafter, the cells were hybridized with RNAscope Multiplex FL V2 AMP1 for 30 minutes, AMP2 for 30 minutes, and AMP3 (Advanced Cell Diagnostic) for 15 minutes at $40^{\circ} \mathrm{C}$. Afterward, the cells were stained with fluorophores (Opal 570, Opal 620, Opal 520, PerkinElmer) followed by HRP incubation (HRP-C1-Opal 570, HPR-C2-Opal 620, HPR-C3-Opal 520 [Advanced Cell Diagnostic]). Between each staining, the HRP Blocker (Advanced Cell Diagnostic) was used to terminate the reaction. After the last HRP block, the slides were washed twice with $1 \times$ wash buffer (Advanced Cell Diagnostic), and DAPI (Advanced Cell Diagnostic) was added for 30 seconds at room temperature. Finally, the slides were mounted using ProLong Gold Antifade Mountant (Thermo Fisher Scientific) and stored at $4^{\circ} \mathrm{C}$ until imaging.

The resulting hybridized slides were imaged using a LSM780 confocal microscope (Zeiss) and analyzed by NIH ImageJ software. DAPI-stained F4/80+ IMS macrophages expressing mRNA of Il-1 $\beta$, Arg-1, Penk, Il-1 $/ \mathrm{Arg}-1, \mathrm{Il}-1 \beta / \mathrm{Penk}$, or Arg-1/Penk were quantified manually in a blind manner (see above for blinding). Four slides representing 4 independent biological samples per condition (vehicle, IL-4) were analyzed. Two fields (each $0.45 \mathrm{~mm}^{2}$ ) per slide were chosen randomly, considering that the fields were not empty or did not contain too many clumped cells, and analyzed using $20 \times$ objective. The number of cells expressing the above-listed mRNAs from the 2 fields (each containing a total of 122-341 cells) was averaged per slide. The data are expressed as the percentage of macrophages expressing the mRNA of interest, by dividing the number of macrophages expressing the mRNA of interest by the total number of macrophages, multiplied by 100 .

Statistics. Statistical analyses were performed using GraphPad Prism software (version 5.02 for Windows; GraphPad Software Inc.). Normality of the data distribution was assessed by D'Agostino-Pearson or Kolmogorov-Smirnov tests. Grubbs' test was performed to identify outliers. No animals or samples were excluded from the analysis. Over time, two groups in the in vivo experiments were compared by 2-way repeated-measures ANOVA followed by Bonferroni's multiple comparison test. In ex vivo experiments, two-sample comparisons were made with the 2-tailed $t$ test for independent, normally distributed data or Mann-Whitney $U$ test for independent, nonnormally distributed data. Multiple comparisons were performed by 1-way repeated-measures ANOVA followed by Bonferroni's multiple comparison test. The data are expressed as individual data points and/or mean \pm SEM. Differences were considered significant at values of $P<0.05$.

Study approval. Experiments were approved by the state animal care committee (Landesamt für Gesundheit und Soziales, Berlin, Germany) and were performed according to the ARRIVE guidelines (69) and NIH Guide for the Care and Use of Laboratory Animals (National Academies Press, 2011).

\section{Author contributions}

MÖC planned and carried out ex vivo experiments; acquired, analyzed, and interpreted the data (Figures 2-6 and Supplemental Figure 3); and wrote the paper. DL planned and carried out in vivo experiments; acquired, analyzed, and interpreted the data (Figures 1, 7, and 8 and Supplemental Figures 1, 2, and 4); performed nerve injury for all ex vivo experiments; and helped write the paper. MÖC and DL are assigned as equally contributing first authors following their mutual decision. JK gave support in planning and performing flow cytometry and IMS experiments. RG gave support in planning and performing flow cytometry experiments. HM conceptualized and supervised the project, designed experiments, interpreted the results, and wrote the paper. All authors read and approved the final version of the manuscript.

\section{Acknowledgments}

We thank Morgane Rouault (Advanced Cell Diagnostic) for help planning and performing the RNAscope experiments. This study was supported by a grant from the Deutsche Forschungsgemeinschaft (MA $6432 / 2-1$ to HM). 
Address correspondence to: Halina Machelska, Department of Experimental Anesthesiology, Charité - Universitätsmedizin Berlin, Campus Benjamin Franklin, Berlin, Germany. Phone: 49.30.450.551677; Email: halina.machelska@charite.de.

JK's present address is: Berlin Institute of Health Cytometry Core, Charité - Universitätsmedizin Berlin, Campus Virchow Klinikum, Berlin, Germany.

1. Wills-Karp M, Finkelman FD. Untangling the complex web of IL-4- and IL-13-mediated signaling pathways. Sci Signal. 2008;1(51):pe55.

2. Gadani SP, Cronk JC, Norris GT, Kipnis J. IL-4 in the brain: a cytokine to remember. J Immunol. 2012;189(9):4213-4219.

3. Cunha FQ, Poole S, Lorenzetti BB, Veiga FH, Ferreira SH. Cytokine-mediated inflammatory hyperalgesia limited by interleukin-4. Br J Pharmacol. 1999;126(1):45-50.

4. Vale ML, et al. Antinociceptive effects of interleukin-4, -10 , and -13 on the writhing response in mice and zymosan-induced knee joint incapacitation in rats. J Pharmacol Exp Ther. 2003;304(1):102-108.

5. Hao S, Mata M, Glorioso JC, Fink DJ. HSV-mediated expression of interleukin-4 in dorsal root ganglion neurons reduces neuropathic pain. Mol Pain. 2006;2:6.

6. Machelska H. Dual peripheral actions of immune cells in neuropathic pain. Arch Immunol Ther Exp (Warsz). 2011;59(1):11-24.

7. Calvo M, Dawes JM, Bennett DL. The role of the immune system in the generation of neuropathic pain. Lancet Neurol. 2012;11(7):629-642

8. Ji RR, Chamessian A, Zhang YQ. Pain regulation by non-neuronal cells and inflammation. Science. 2016;354(6312):572-577.

9. Sica A, Mantovani A. Macrophage plasticity and polarization: in vivo veritas. J Clin Invest. 2012;122(3):787-795.

10. Hu X, et al. Microglial and macrophage polarization - new prospects for brain repair. Nat Rev Neurol. 2015;11(1):56-64.

11. Uçeyler N, Schäfers M, Sommer C. Mode of action of cytokines on nociceptive neurons. Exp Brain Res. 2009;196(1):67-78.

12. Kiguchi N, Kobayashi Y, Saika F, Sakaguchi H, Maeda T, Kishioka S. Peripheral interleukin-4 ameliorates inflammatory macrophage-dependent neuropathic pain. Pain. 2015;156(4):684-693.

13. Stein C, Hassan AH, Przewłocki R, Gramsch C, Peter K, Herz A. Opioids from immunocytes interact with receptors on sensory nerves to inhibit nociception in inflammation. Proc Natl Acad Sci U S A. 1990;87(15):5935-5939.

14. Stein C, Hassan AH, Lehrberger K, Giefing J, Yassouridis A. Local analgesic effect of endogenous opioid peptides. Lancet. 1993;342(8867):321-324.

15. Rittner HL, et al. Pain control by CXCR2 ligands through $\mathrm{Ca}^{2+}$-regulated release of opioid peptides from polymorphonuclear cells. FASEB J. 2006;20(14):2627-2629.

16. Labuz D, Schmidt Y, Schreiter A, Rittner HL, Mousa SA, Machelska H. Immune cell-derived opioids protect against neuropathic pain in mice. J Clin Invest. 2009;119(2):278-286.

17. Stein C, Machelska H. Modulation of peripheral sensory neurons by the immune system: implications for pain therapy. Pharmacol Rev. 2011;63(4):860-881.

18. Celik MÖ, et al. Leukocyte opioid receptors mediate analgesia via $\mathrm{Ca}(2+)$-regulated release of opioid peptides. Brain Behav Immun. 2016;57:227-242.

19. Pannell M, et al. Adoptive transfer of M2 macrophages reduces neuropathic pain via opioid peptides. J Neuroinflammation. 2016;13(1):262.

20. Bennett GJ, Xie YK. A peripheral mononeuropathy in rat that produces disorders of pain sensation like those seen in man. Pain. 1988;33(1):87-107.

21. Moalem G, Tracey DJ. Immune and inflammatory mechanisms in neuropathic pain. Brain Res Rev. 2006;51(2):240-264.

22. Massier J, Eitner A, Banchet GS von, Schaible H-G. Effects of differently activated rodent macrophages on sensory neurons: implications for arthritis pain. Arthritis Rheumatol. 2015;67(8):2263-2272.

23. Chen P, Piao X, Bonaldo P. Role of macrophages in Wallerian degeneration and axonal regeneration after peripheral nerve injury. Acta Neuropathol. 2015;130(5):605-618.

24. Liu T, van Rooijen N, Tracey DJ. Depletion of macrophages reduces axonal degeneration and hyperalgesia following nerve injury. Pain. 2000;86(1-2):25-32.

25. Rutkowski MD, Pahl JL, Sweitzer S, van Rooijen N, DeLeo JA. Limited role of macrophages in generation of nerve injury-induced mechanical allodynia. Physiol Behav. 2000;71(3-4):225-235.

26. Brack A, et al. Tissue monocytes/macrophages in inflammation: hyperalgesia versus opioid-mediated peripheral antinociception. Anesthesiology. 2004;101(1):204-211.

27. Barclay J, et al. Role of the cysteine protease cathepsin S in neuropathic hyperalgesia. Pain. 2007;130(3):225-234.

28. Godai K, et al. Peripheral administration of morphine attenuates postincisional pain by regulating macrophage polarization through COX-2-dependent pathway. Mol Pain. 2014;10:36.

29. Mosser DM, Edwards JP. Exploring the full spectrum of macrophage activation. Nat Rev Immunol. 2008;8(12):958-969

30. Van Dyken SJ, Locksley RM. Interleukin-4- and interleukin-13-mediated alternatively activated macrophages: roles in homeostasis and disease. Annu Rev Immunol. 2013;31:317-343.

31. Hickey MJ, Granger DN, Kubes P. Molecular mechanisms underlying IL-4-induced leukocyte recruitment in vivo: a critical role for the alpha 4 integrin. J Immunol. 1999;163(6):3441-3448.

32. Ratthé C, Ennaciri J, Garcês Gonçalves DM, Chiasson S, Girard D. Interleukin (IL)-4 induces leukocyte infiltration in vivo by an indirect mechanism. Mediators Inflamm. 2009;2009:193970.

33. Jenkins SJ, et al. Local macrophage proliferation, rather than recruitment from the blood, is a signature of TH2 inflammation. Science. 2011;332(6035):1284-1288. 
34. Brown DR, Goldberg LI. The use of quaternary narcotic antagonists in opiate research. Neuropharmacology. 1985;24(3):181-191.

35. Spahn V, et al. A nontoxic pain killer designed by modeling of pathological receptor conformations. Science. 2017;355(6328):966-969.

36. Machelska H, Celik MÖ. Advances in achieving opioid analgesia without side effects. Front Pharmacol. 2018;9:1388

37. Gordon S, Martinez FO. Alternative activation of macrophages: mechanism and functions. Immunity. 2010;32(5):593-604.

38. Oetjen LK, et al. Sensory neurons co-opt classical immune signaling pathways to mediate chronic itch. Cell. 2017;171(1):217-228.e13.

39. Vogelaar CF, et al. Fast direct neuronal signaling via the IL-4 receptor as therapeutic target in neuroinflammation. Sci Transl Med. 2018;10(430):eaao2304.

40. Truong W, Cheng C, Xu QG, Li XQ, Zochodne DW. Mu opioid receptors and analgesia at the site of a peripheral nerve injury. Ann Neurol. 2003;53(3):366-375.

41. Kabli N, Cahill CM. Anti-allodynic effects of peripheral delta opioid receptors in neuropathic pain. Pain. 2007;127(1-2):84-93.

42. Schmidt Y, Gavériaux-Ruff C, Machelska H. $\mu$-Opioid receptor antibody reveals tissue-dependent specific staining and increased neuronal $\mu$-receptor immunoreactivity at the injured nerve trunk in mice. PLoS One. 2013;8(11):e79099.

43. Guilbaud G, Gautron M, Jazat F, Ratinahirana H, Hassig R, Hauw JJ. Time course of degeneration and regeneration of myelinated nerve fibres following chronic loose ligatures of the rat sciatic nerve: can nerve lesions be linked to the abnormal pain-related behaviours? Pain. 1993;53(2):147-158.

44. Schäfer MK, Bette M, Romeo H, Schwaeble W, Weihe E. Localization of kappa-opioid receptor mRNA in neuronal subpopulations of rat sensory ganglia and spinal cord. Neurosci Lett. 1994;167(1-2):137-140.

45. Minami M, Maekawa K, Yabuuchi K, Satoh M. Double in situ hybridization study on coexistence of $\mu$-, $\delta$-, and $\kappa$-opioid receptor mRNAs with preprotachykinin A mRNA in the rat dorsal root ganglia. Brain Res Mol Brain Res. 1995;30(2):203-210.

46. Gaveriaux-Ruff C, et al. Genetic ablation of $\delta$ opioid receptors in nociceptive sensory neurons increases chronic pain and abolishes opioid analgesia. Pain. 2011;152(6):1238-1248.

47. Weibel R, et al. Mu opioid receptors on primary afferent nav1.8 neurons contribute to opiate-induced analgesia: insight from conditional knockout mice. PLoS One. 2013;8(9):e74706.

48. Koltzenburg M, Lundberg LE, Torebjörk HE. Dynamic and static components of mechanical hyperalgesia in human hairy skin. Pain. 1992;51(2):207-219.

49. Field MJ, Bramwell S, Hughes J, Singh L. Detection of static and dynamic components of mechanical allodynia in rat models of neuropathic pain: are they signalled by distinct primary sensory neurones? Pain. 1999;83(2):303-311.

50. Cayla C, Labuz D, Machelska H, Bader M, Schäfer M, Stein C. Impaired nociception and peripheral opioid antinociception in mice lacking both kinin B1 and B2 receptors. Anesthesiology. 2012;116(2):448-457.

51. Labuz D, Machelska H. Stronger antinociceptive efficacy of opioids at the injured nerve trunk than at its peripheral terminals in neuropathic pain. J Pharmacol Exp Ther. 2013;346(3):535-544.

52. Rodriguez-Gaztelumendi A, Spahn V, Labuz D, Machelska H, Stein C. Analgesic effects of a novel pH-dependent $\mu$-opioid receptor agonist in models of neuropathic and abdominal pain. Pain. 2018;159(11):2277-2284.

53. Labuz D, Celik MÖ, Zimmer A, Machelska H. Distinct roles of exogenous opioid agonists and endogenous opioid peptides in the peripheral control of neuropathy-triggered heat pain. Sci Rep. 2016;6:32799.

54. Hasegawa-Moriyama M, et al. Peroxisome proliferator-activated receptor-gamma agonist rosiglitazone attenuates inflammatory pain through the induction of heme oxygenase-1 in macrophages. Pain. 2013;154(8):1402-1412.

55. Üçeyler N, Topuzoğlu T, Schiesser P, Hahnenkamp S, Sommer C. IL-4 deficiency is associated with mechanical hypersensitivity in mice. PLoS One. 2011;6(12):e28205.

56. Luo X, et al. Macrophage toll-like receptor 9 contributes to chemotherapy-induced neuropathic pain in male mice. $J$ Neurosci. 2019;39(35):6848-6864.

57. Grace PM, Hutchinson MR, Maier SF, Watkins LR. Pathological pain and the neuroimmune interface. Nat Rev Immunol. 2014;14(4):217-231.

58. Busch-Dienstfertig M, González-Rodríguez S. IL-4, JAK-STAT signaling, and pain. JAKSTAT. 2013;2(4):e27638.

59. Uçeyler N, Valenza R, Stock M, Schedel R, Sprotte G, Sommer C. Reduced levels of antiinflammatory cytokines in patients with chronic widespread pain. Arthritis Rheum. 2006;54(8):2656-2664.

60. Uçeyler N, Rogausch JP, Toyka KV, Sommer C. Differential expression of cytokines in painful and painless neuropathies. Neurology. 2007;69(1):42-49.

61. Ghoreschi K, et al. Interleukin-4 therapy of psoriasis induces Th2 responses and improves human autoimmune disease. Nat Med. 2003;9(1):40-46.

62. Roberti ML, et al. Immunomodulating treatment with low dose interleukin-4, interleukin-10 and interleukin-11 in psoriasis vulgaris. J Biol Regul Homeost Agents. 2014;28(1):133-139.

63. Martin R. Interleukin 4 treatment of psoriasis: are pleiotropic cytokines suitable therapies for autoimmune diseases? Trends Pharmacol Sci. 2003;24(12):613-616.

64. Chaplan SR, Bach FW, Pogrel JW, Chung JM, Yaksh TL. Quantitative assessment of tactile allodynia in the rat paw. J Neurosci Methods. 1994;53(1):55-63.

65. Cossarizza A, et al. Guidelines for the use of flow cytometry and cell sorting in immunological studies. Eur J Immunol. 2017;47(10):1584-1797.

66. Roederer M. Compensation in flow cytometry. Curr Protoc Cytom. 2002; Chapter 1:Unit 1.14.

67. Mittag A, Tárnok A. Basics of standardization and calibration in cytometry--a review. J Biophotonics. 2009;2(8-9):470-481.

68. Wang F, et al. RNAscope: a novel in situ RNA analysis platform for formalin-fixed, paraffin-embedded tissues. JMol Diagn. 2012;14(1):22-29.

69. Kilkenny C, Browne WJ, Cuthill IC, Emerson M, Altman DG. Improving bioscience research reporting: the ARRIVE guidelines for reporting animal research. PLoS Biol. 2010;8(6):e1000412. 